\title{
THE LIST DISTINGUISHING NUMBER EQUALS THE DISTINGUISHING NUMBER FOR INTERVAL GRAPHS
}

\author{
Poppy Immel and Paul S. Wenger \\ School of Mathematical Sciences \\ Rochester Institute of Technology \\ Rochester, NY, USA \\ e-mail: pgi8114@rit.edu \\ pswsma@rit.edu
}

\begin{abstract}
A distinguishing coloring of a graph $G$ is a coloring of the vertices so that every nontrivial automorphism of $G$ maps some vertex to a vertex with a different color. The distinguishing number of $G$ is the minimum $k$ such that $G$ has a distinguishing coloring where each vertex is assigned a color from $\{1, \ldots, k\}$. A list assignment to $G$ is an assignment $L=\{L(v)\}_{v \in V(G)}$ of lists of colors to the vertices of $G$. A distinguishing $L$-coloring of $G$ is a distinguishing coloring of $G$ where the color of each vertex $v$ comes from $L(v)$. The list distinguishing number of $G$ is the minimum $k$ such that every list assignment to $G$ in which $|L(v)|=k$ for all $v \in V(G)$ yields a distinguishing $L$-coloring of $G$. We prove that if $G$ is an interval graph, then its distinguishing number and list distinguishing number are equal.
\end{abstract}

Keywords: distinguishing, distinguishing number, list distinguishing, interval graph.

2010 Mathematics Subject Classification: 05C60.

\section{REFERENCES}

[1] M.O. Albertson, Distinguishing Cartesian powers of graphs, Electron. J. Combin. 12 (2005) \#N17.

[2] M.O. Albertson and K.L. Collins, Symmetry breaking in graphs, Electron. J. Combin. 3 (1996) \#R18.

[3] V. Arvind, C. Cheng and N. Devanur, On computing the distinguishing numbers of planar graphs and beyond: a counting approach, SIAM J. Discrete Math. 22 (2008) 1297-1324.

doi:10.1137/07068686X 
[4] V. Arvind and N. Devanur, Symmetry breaking in trees and planar graphs by vertex coloring, in: Proceedings of the 8th Nordic Combinatorial Conference, Aalborg University (Aalborg, Denmark, 2004).

[5] K. Booth and G. Lueker, Testing for the consecutive ones property, interval graphs, and graph planarity using PQ-tree algorithms, J. Comput. System Sci. 13 (1976) 335-379. doi:10.1016/S0022-0000(76)80045-1

[6] C.T. Cheng, On computing the distinguishing and distinguishing chromatic numbers of interval graphs and other results, Discrete Math. 309 (2009) 5169-5182. doi:10.1016/j.disc.2009.04.004

[7] C.T. Cheng, On computing the distinguishing numbers of trees and forests, Electron. J. Combin. 13 (2006) \#R11.

[8] C. Colbourn and K. Booth, Linear time automorphism algorithms for trees, interval graphs, and planar graphs, SIAM J. Comput. 10 (1981) 203-225. doi:10.1137/0210015

[9] P. Erdős, A. Rubin and H. Taylor, Choosability in graphs, Congr. Numer. 26 (1980) $125-157$.

[10] M. Ferrara, B. Flesch and E. Gethner, List-distinguishing colorings of graphs, Electron. J. Combin. 18 (2011) \#P161.

[11] M. Ferrara, E. Gethner, S.G. Hartke, D. Stolee and P.S. Wenger, List distinguishing parameters of trees, Discrete Appl. Math. 161 (2013) 864-869. doi:10.1016/j.dam.2012.10.003

[12] M. Ferrara, E. Gethner, S.G. Hartke, D. Stolee and P.S. Wenger, Extending precolorings to distinguish group actions, arXiv:1405.5558 [math.CO].

[13] M. Fisher and G. Isaak, Distinguishing colorings of Cartesian products of complete graphs, Discrete Math. 308 (2008) 2240-2246. doi:10.1016/j.disc.2007.04.070

[14] D. Fulkerson and O. Gross, Incidence matrices and interval graphs, Pacific J. Math. 15 (1965) 835-855.

[15] W. Imrich, J. Jerebic and S. Klavžar, The distinguishing number of Cartesian products of complete graphs, European J. Combin. 29 (2008) 922-929. doi:10.1016/j.ejc.2007.11.018

[16] W. Imrich and S. Klavžar, Distinguishing Cartesian powers of graphs, J. Graph Theory 53 (2006) 250-260. doi:10.1002/jgt.20190

[17] W. Imrich, S. Klavžar and V. Trofimov, Distinguishing infinite graphs, Electron. J. Combin. 14 (2007) \#R36.

[18] S. Klavžar, T.-L. Wong and X. Zhu, Distinguishing labellings of group action on vector spaces and graphs, J. Algebra 303 (2006) 626-641. doi:10.1016/j.jalgebra.2006.01.045 
[19] S. Klavžar and X. Zhu, Cartesian powers of graphs can be distinguished by two labels, European J. Combin. 28 (2007) 303-310.

doi:10.1016/j.ejc.2005.07.001

[20] A. Lombardi, Distinguishing extension numbers for $\mathbb{R}^{n}$ and $S^{n}$, arXiv:1408.5849 [math.co] (2014).

[21] G. Lueker and K. Booth, A linear time algorithm for deciding interval graph isomorphism, J. Assoc. Comput. Mach. 26 (1979) 183-195. doi: $10.1145 / 322123.322125$

[22] V.G. Vizing, Coloring the vertices of a graph in prescribed colors, Diskret. Analiz, Metody Diskret. Anal. v Teorii Kodov i Shem 29 (1976) 3-10 (in Russian).

Received 15 September 2015

Revised 23 March 2016

Accepted 23 March 2016 